\title{
How are the Locations of Objects in the Environment Represented in Memory?
}

\author{
Timothy P. McNamara ${ }^{1}$ \\ Department of Psychology, Vanderbilt University, $11121^{\text {st }}$ Ave South \\ Nashville, TN 37203 \\ t.mcnamara@vanderbilt.edu
}

\begin{abstract}
This chapter summarizes a new theory of spatial memory. According to the theory, when people learn the locations of objects in a new environment, they interpret the spatial structure of that environment in terms of a spatial reference system. Our current conjecture is that a reference system intrinsic to the collection of objects is used. Intrinsic axes or directions are selected using egocentric (e.g., viewing perspective) and environmental (e.g., walls of the surrounding room) cues. The dominant cue is egocentric experience. The reference system selected at the first view is typically not updated with additional views or observer movement. However, if the first view is misaligned but a subsequent view is aligned with natural and salient axes in the environment, a new reference system is selected and the layout is reinterpreted in terms of this new reference system. The chapter also reviews evidence on the orientation dependence of spatial memories and recent results indicating that two representations may be formed when people learn a new environment; one preserves interobject spatial relations and the other comprises visual memories of experienced views.
\end{abstract}

\section{Introduction}

As any student of spatial cognition or geography knows, the concept of location is inherently relative. One cannot describe or specify the location of an object without providing, at least implicitly, a frame of reference. For example, the location of a chair in a classroom can be specified in terms of the room itself (e.g., the chair is in the corner by the door), other chairs in the room (e.g., the chair is in the first row, second column), or an observer (e.g., the chair is in front of me). Likewise, human memory systems must use spatial reference systems of some kind to preserve the remembered locations of objects.

There are many ways to classify spatial reference systems (Levinson, 1996), but a useful one, for the purposes of understanding human spatial memory, divides them into two categories: Egocentric reference systems specify location and orientation with respect to the organism, and include eye, head, and body coordinates. Environmental reference systems specify location and orientation with respect to

\footnotetext{
${ }^{1}$ Preparation of this chapter and the research reported in it were supported in part by National Institute of Mental Health Grant R01-MH57868. The chapter was improved as a result of the comments of two anonymous reviewers. I am enormously indebted to Vaibhav Diwadkar, Weimin Mou, Björn Rump, Amy Shelton, Christine Valiquette, and Steffen Werner for their contributions to the empirical and theoretical developments summarized in this chapter.
} 
elements and features of the environment, such as the perceived direction of gravity, landmarks, or the floor, ceiling, and walls of a room.

The initial investigations of spatial reference systems conducted in our laboratory indicated that spatial memories might be defined egocentrically (e.g., Diwadkar \& McNamara, 1997; Roskos-Ewoldsen, McNamara, Shelton, \& Carr, 1998; Shelton \& McNamara, 1997). For example, Shelton and McNamara (1997) required participants to learn the locations of seven objects in a room from two orthogonal viewpoints. After they had memorized the locations of the objects, the observers were escorted to a different room, on a different floor of the building, and asked to make judgments of relative direction using their memories (e.g., "Imagine you are standing at the shoe and facing the clock. Point to the jar."). These judgments were made with a computer mouse on a simulated dial and pointer displayed on the computer screen. Pointing judgments were faster and more accurate for imagined headings parallel to one of the two study views than for headings parallel to unfamiliar views. These results suggested that participants had formed two egocentric representations of the layout, one from each viewing position. We conceived of these representations as visualspatial "snapshots" of the layout.

The results of subsequent investigations indicated that this conclusion was premature. Werner and Schmidt (1999) asked student residents of Göttingen, Germany to imagine themselves at the intersection of two major streets in town, facing in various directions, and then to identify landmarks in cued directions. They found that landmarks were identified faster and more accurately when the imagined heading was parallel to one of the major streets than when it was not (see also, Montello, 1991). This finding indicates that the students had represented the layout of the city in terms of reference axes established by the road grid.

More problematic still are results of experiments reported by Shelton and McNamara (2001b). In Shelton and McNamara's third experiment, participants learned the locations of objects in a room from two stationary points of view. One viewing position was aligned $\left(0^{\circ}\right)$ and the other was misaligned $\left(135^{\circ}\right)$ with a mat on the floor and the walls of the room (see Figure 1). Performance in subsequent judgments of relative direction indicated that the aligned view was represented in memory but the misaligned view was not (see Figure 2). Note that angular error in pointing judgments was as high for the familiar heading of $135^{\circ}$ as for unfamiliar headings, even for participants who learned the view from $135^{\circ}$ first! In another experiment, participants learned similar layouts in a cylindrical room from three points of view $\left(0^{\circ}, 90^{\circ}, \& 225^{\circ}\right)$. Half of the participants learned the views in the order $0^{\circ}-90^{\circ}-225^{\circ}$, and half learned the views in the reverse order. Accuracy of judgments of relative direction indicated that only the first study view $\left(0^{\circ}\right.$ or $\left.225^{\circ}\right)$ was mentally represented: Pointing judgments were quite accurate for imagined headings parallel to the first study view (mean error of $14.6^{\circ}$ ) but no more accurate for the second and third study views than for novel headings (mean error of $38.7^{\circ} \mathrm{vs}$. $35.7^{\circ}$, respectively).

The visual-spatial snapshot model proposed by Shelton and McNamara (1997) would predict better performance on familiar than on unfamiliar headings. For example, in the cylindrical room experiment, it predicts, ceteris paribus, equally good performance on the headings of $0^{\circ}, 90^{\circ}$, and $225^{\circ}$. The results of Werner and Schmidt's (1999) and Shelton and McNamara's (2001b) experiments indicated that 
spatial memories were not egocentric, and led to the development of the theory of spatial memory described in the next section.

\section{Sketch of a Theory of Human Spatial Memory}

The theory of spatial memory that we have developed to explain these findings is firmly rooted in principles of form perception proposed by Rock (1973). Rock wanted to know why the perceived shape of a figure depends on its orientation. A square, for example, is seen as a square when an edge is on top, but is seen as a diamond when a vertex is on top. Rock was particularly interested in whether a

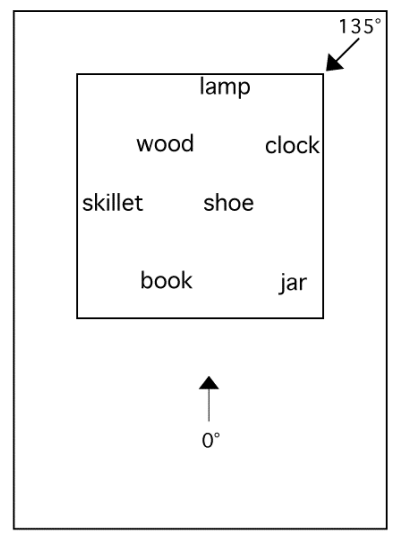

Figure 1. Schematic illustration of one of the layouts used in Shelton and McNamara's (2001b) Experiment 3. Real objects were used, not names.

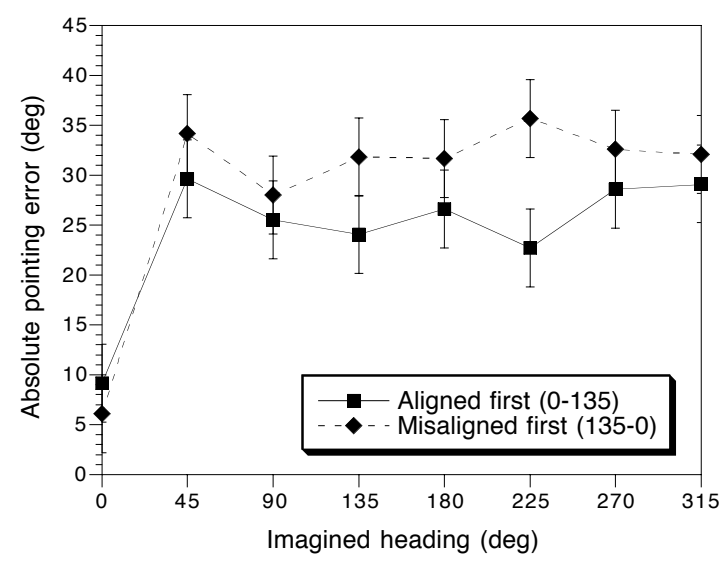

Figure 2. Angular error in judgments of relative direction as a function of imagined heading and the order in which views were learned in Shelton and McNamara's (2001b) Experiment 3. Subjects learned an aligned view $\left(0^{\circ}\right)$ and a misaligned view $\left(135^{\circ}\right)$ of layouts similar to the one illustrated in Figure 1. Error bars are confidence intervals corresponding to \pm 1 SEM as estimated from the ANOVA. 
change in orientation with respect to the observer or a change in orientation with respect to the environment was the principal cause of changes in perceived shape.

Rock's investigations indicated that for unfamiliar figures, changing egocentric orientation had little effect on perceived shape. However, when the orientation of a figure with respect to the environment was changed, the figure was seen as different and often not recognized at all. For example, Rock (1956) designed ambiguous figures so that they had different interpretations in different orientations; for instance, in one orientation, one of the figures looked like the profile of an old man, but when rotated 90 degrees, it looked like an outline of the U.S. The figures were presented to observers whose heads were tilted 90 degrees. When shown these ambiguous figures with heads tilted, observers typically reported seeing the environmentally upright figure rather than the retinally upright figure. Another way to describe these findings is that observers saw the shape defined by the environmental frame of reference rather than the shape defined by the egocentric frame of reference; indeed, they ignored the egocentric information to interpret the figure in terms of the environmental information.

Rock (1973) concluded that the interpretation of a figure depends on which part or region is assigned "top," and that a change in the assignment of this direction profoundly affects perceived shape. The top of a figure is normally assigned on the basis of the information provided by gravity or the visual frame of reference. Other sources of information can also be used, including egocentric orientation, instructions, intrinsic properties of the figure, and familiarity, but these sources were, according to Rock, typically less salient than environmental sources. More recent investigations (e.g., Friedman \& Hall, 1996; McMullen \& Jolicoeur, 1990) have shown that Rock might have underestimated the importance of retinal orientation in the perception of form. Even so, the general principle--that the perception of form involves the assignment of directions based on a spatial reference system--is sound.

According to our theory (Mou \& McNamara, 2002; Shelton \& McNamara, 2001b; Werner \& Schmidt, 1999), learning the spatial structure of a new environment involves interpreting it in terms of a spatial reference system. This process is analogous to determining the top of a figure or an object; in effect, conceptual "north" is assigned to the layout, creating privileged directions in the environment (conceptual "north" need not, and usually will not, correspond to true or magnetic north or any other cardinal direction). Our working hypothesis is that the spatial structure of the environment is represented in terms of an intrinsic reference system (Palmer, 1989); one defined by the layout itself (e.g., the rows and columns formed by chairs in a classroom). Intrinsic directions or axes are selected using cues, such as viewing perspective and other experiences (e.g., instructions), properties of the objects (e.g., they may be grouped together based on similarity or proximity), and the structure of the environment (e.g., geographical slant). An important difference between form perception and spatial memory is that whereas figures in the frontal plane are oriented in a space with a powerful reference axis, namely, gravity, the locations of objects are typically defined in the ground plane, which does not have privileged axes or directions (e.g., humans cannot perceive magnetic fields). We therefore propose that the dominant cue in spatial memory is egocentric experience. The spatial layouts learned by participants in most of our experiments were composed of small, moveable objects. In general, however, a spatial layout could be composed of large or 
stationary objects, such as mountain peaks, trees, buildings, doors, windows, and so forth. We would still expect in such cases for intrinsic directions or axes to be identifiable, and for some to be more salient than others.

The theory is perhaps best understood in the context of concrete examples. Consider, first, the cylindrical room experiment conducted by Shelton and McNamara (2001b, Exp. 7). According to the theory, when observers studied the layout from the first viewing position, they interpreted its spatial structure in terms of an intrinsic reference system aligned with their viewing perspective. When participants were taken to the second and third points of view, they continued to interpret the spatial structure of the layout in terms of the reference system selected at the first point of view, just as if they were viewing a (now) familiar object at novel orientations. This reference system remained the dominant one, even when participants were moved to the next two points of view, because the layout did not have salient alternative axes, and because no other point of view was aligned with a salient axis in the environment.

As another example, consider the experiment summarized above in which participants learned an aligned and a misaligned view of a layout of objects (Figures 1 \& 2). Participants who first learned the aligned viewpoint $\left(0^{\circ}\right)$ represented the layout in terms of an intrinsic reference system aligned with their viewing perspective, the edges of the mat, and the walls of the room. When they moved to the misaligned viewpoint $\left(135^{\circ}\right)$, they still interpreted the layout in terms of the reference system established by the first, aligned view. Hence, performance in judgments of relative direction was best for the heading parallel to the aligned view, and was no better for the heading parallel to the misaligned view than for novel headings. Observers who first learned the misaligned view $\left(135^{\circ}\right)$ also must have interpreted the space in terms of a reference system defined by that view. This conclusion follows from the results of another experiment in which participants learned the same layout but only from the misaligned point of view (Shelton \& McNamara, 2001b, Exp. 2). The results of this experiment showed that participants represented the layout from this single familiar view. Our hypothesis is that when participants were taken to the second, aligned viewpoint, they re-interpreted the spatial structure of the layout in terms of a reference system defined by the aligned view because it was aligned with salient axes in the environment (e.g., the edges of the mat and the walls of the room) and with egocentric experience (albeit, a new experience). After moving from a misaligned study view to an aligned study view, observers changed the definition of "north." A new spatial reference system--one that was aligned with the environment and egocentric experience--was selected and the spatial layout was reinterpreted in terms of it.

Our conjecture that spatial memories are defined in terms of intrinsic reference systems is supported by findings reported by Mou and McNamara (2002). They required participants to learn layouts like the one illustrated in Figure 3. Objects were placed on a square mat oriented with the walls of the enclosing room or on the bare floor of a cylindrical room. In one experiment, participants studied the layout from $315^{\circ}$, and were instructed to learn the layout along the egocentric $315^{\circ}$ axis or the nonegocentric $0^{\circ}$ axis. This instructional manipulation was accomplished by pointing out that the layout could be seen in "columns" consistent with the appropriate axis (e.g., clock-jar, scissors-shoe, etc. vs. scissors-clock, wood-shoe-jar, etc.), and by asking participants to point to the objects in the appropriate order when they were 
quizzed during the learning phase. All participants viewed the layout from $315^{\circ}$. After learning, participants made judgments of relative direction using their memory of the layout.

One important result (see Figure 4) is the near perfect crossover interaction for imagined headings of $0^{\circ}$ and $315^{\circ}$ : Participants who were instructed to learn the layout along the egocentric $315^{\circ}$ axis were better able to imagine the spatial structure of the layout from the $315^{\circ}$ heading than from the $0^{\circ}$ heading, whereas the opposite pattern was obtained for participants who learned the layout along the nonegocentric $0^{\circ}$ axis. In particular, participants in the $0^{\circ}$ group were better able to imagine the

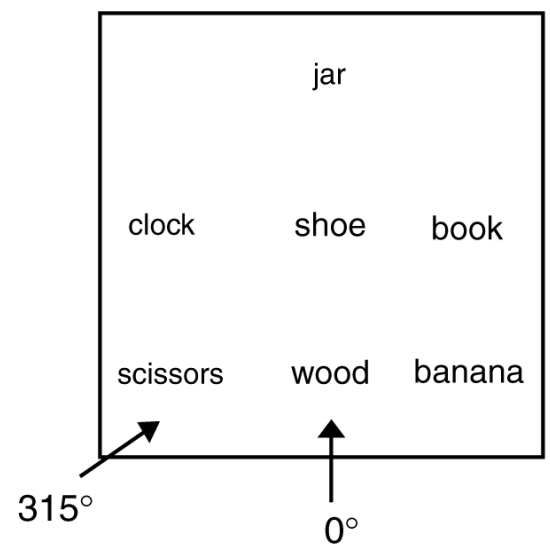

Figure 3. Schematic illustration of one of the layouts used by Mou and McNamara (2002). Real objects were used, not names.

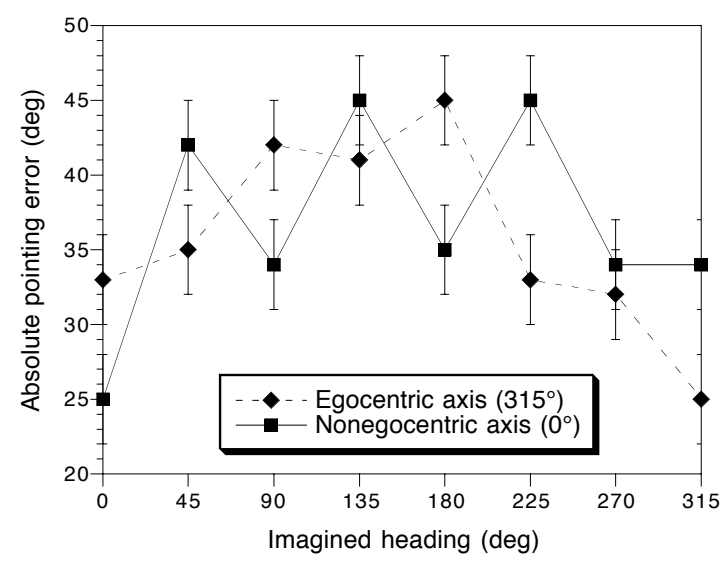

Figure 4. Angular error in judgments of relative direction as a function of imagined heading and learning axis in Mou and McNamara's (2002) Experiment 2. All subjects viewed the layout in Figure 3 from $315^{\circ}$. They were instructed to learn the layout along the egocentric $315^{\circ}-135^{\circ}$ axis or the nonegocentric $0^{\circ}-180^{\circ}$ axis. Error bars are confidence intervals corresponding to $\pm 1 \mathrm{SEM}$ as estimated from the ANOVA. 
spatial structure of the layout from an unfamiliar heading $\left(0^{\circ}\right)$ than from the heading they actually experienced $\left(315^{\circ}\right)$. A second important finding is the different patterns of results for the two groups: In the $0^{\circ}$ group, performance was better on novel headings orthogonal or opposite to $0^{\circ}\left(90^{\circ}, 180^{\circ}, \& 270^{\circ}\right)$ than on other novel headings, producing a sawtooth pattern, whereas in the $315^{\circ}$ group performance on novel headings depended primarily on the angular distance to the familiar heading of $315^{\circ}$. The sawtooth pattern in the $0^{\circ}$ group also appeared when the objects were placed on the bare floor of a cylindrical room, which indicates that this pattern was produced by the intrinsic structure of the layout, not by the mat or the walls of the enclosing room. The third major finding was that there was no apparent cost to learning the layout from a nonegocentric perspective. Overall error in pointing did not differ across the two groups.

We believe that the sawtooth pattern arises when participants are able to represent the layout along two intrinsic axes (e.g., $0^{\circ}-180^{\circ}$ and $90^{\circ}-270^{\circ}$ ). Performance may be better on the imagined heading of $0^{\circ}$ because this heading was emphasized during the learning phase. We suspect that the sawtooth pattern did not occur in the condition in which participants learned the layout according to the $315^{\circ}$ $135^{\circ}$ axis because the $45^{\circ}-225^{\circ}$ axis is much less salient in the collection of objects. Indeed, we suspect that participants did not usually recognize that the layout could be organized along "diagonal" axes unless they actually experienced them because the "major" axes were much more salient; for example, the layout is bilaterally symmetric around $0^{\circ}-180^{\circ}$ but not around $315^{\circ}-135^{\circ}$ or $45^{\circ}-225^{\circ}$.

\section{Alternative Theories}

Aspects of our theoretical framework have been proposed or anticipated by others. Most notably, Tversky (1981) demonstrated that errors in memory of spatial relations could be explained in terms of heuristics derived from principles of perceptual organization, and argued that spatial memory was influenced by how a map or environment was interpreted when it was learned. She also discussed how intrinsic reference systems might be induced from features of the environment and used to represent location and orientation. Several experiments have demonstrated that spatial representations are influenced by the intrinsic structure of a layout or by the geometry of the surrounding environment (e.g., Easton \& Sholl, 1995; Hermer \& Spelke, 1994; Learmonth, Newcombe, \& Huttenlocher, 2001; Montello, 1991; Werner \& Schmidt, 1999).

Several influential models have been proposed to explain memory for location. Huttenlocher, Hedges, and Duncan (1991) and Lansdale (1998) have proposed elegant mathematical models of positional uncertainty and bias in memory of the location of single object. Neither of these projects was aimed at investigating the spatial reference systems used in memory, although Huttenlocher et al. concluded from the distributions of memory reports that participants used polar coordinates to represent the location of a single dot in a circle. It is not clear how these models could explain the orientation dependence of spatial memories, or how they could be scaled up to large-scale spaces.

The spatial-framework model investigated by Bryant, Franklin, and Tversky (e.g., Bryant \& Tversky, 1999; Franklin \& Tversky, 1990) is more relevant to the 
situations examined in our studies. In particular, Bryant and Tversky (1999) had participants study two-dimensional (2D) diagrams or three-dimensional (3D) models of six objects surrounding a central character in the canonical directions front, back, right, left, head (e.g., above an upright character) and feet (e.g., below an upright character). In the test phase, the participants identified the objects in cued directions. Across trials, the central character was described as rotating to face different objects, and as changing orientation (e.g., from upright to reclining). Bryant and Tversky concluded that diagrams, and other 2D interpretations of the scenes, were represented using an intrinsic reference system centered on the character, whereas the models, and other 3D interpretations of the scenes, were represented with an egocentric spatial framework in which participants mentally adopted the orientation and the facing direction of the central character.

The use of an intrinsic reference system for 2D scenes is broadly consistent with our theoretical framework. As Bryant and Tversky (1999) use the term, it refers to an object-based reference system centered on objects that have intrinsic asymmetries, such as people and cars. In our theoretical framework, it refers to a reference system in which reference directions or axes are induced from the layout of the environment to be learned. The basic idea is similar, however. The egocentric spatial framework used for 3D scenes would seem to be inconsistent with our model. In fact, we believe the two are complementary. Bryant and Tversky's experiments examine situations in which the observer has adopted an orientation in imagination, and then is asked to retrieve objects in cued directions. The difficulty of retrieving or inferring the spatial structure of the layout from novel versus familiar orientations is not measured. Our experiments, in contrast, have focused on effects of orientation, not on the efficiency of retrieval of objects in cued directions. The results of experiments in which both effects have been assessed (e.g., Sholl, 1987; Werner \& Schmidt, 1999) indicate that they may be independent.

The independence of egocentric and allocentric coding of spatial relations is embodied in Sholl's model of spatial representation and retrieval (e.g., Easton \& Sholl, 1995; Sholl \& Nolin, 1997). This model contains two subsystems: The selfreference system codes self-to-object spatial relations in body-centered coordinates, using the body axes of front-back, right-left, and up-down (as in the spatial framework model). This system provides a framework for spatially directed motor activity, such as walking, reaching, and grasping. The object-to-object system codes the spatial relations among objects in environmental coordinates. Spatial relations in this system are specified only with respect to other objects (i.e., an intrinsic reference system is used). Relative direction is preserved locally, among the set of objects, but not with respect to the surrounding environment, and there is no preferred direction or axis. The representation is therefore orientation-independent. These two systems interact in several ways. For example, the heading of the self-reference system fixes the orientation of the object-to-object system, in that the front pole of the front-back axis determines "forward" in the object-to-object system. As the self-reference system changes heading, by way of actual or imagined rotations of the body, the orientation of the object-to-object system changes as well.

At present, our theoretical framework does not address self-to-object spatial relations, although we recognize that such spatial relations must be represented, at least at the perceptual level, for the purpose of guiding action in space and seem to 
play an important role in the spatial-framework paradigm. An important similarity between Sholl's model and ours is the use of intrinsic reference systems to represent interobject spatial relations. A major difference, though, is that the object-to-object system is orientation independent in Sholl's model but orientation dependent in ours.

\section{Orientation Dependence vs. Independence}

Over the past two decades, a large number of experiments have examined, at least indirectly, the orientation dependence of spatial memories. Participants have learned several views of layouts; have learned layouts visually, tactilely, via navigation, and via desktop virtual reality; have been tested in the same room in which they learned the layout or in a different room; have been oriented or disoriented at the time of testing; have been seated or standing during learning and testing; and have been tested using scene recognition, judgments of relative direction, or both (e.g., Christou \& Bülthoff, 1999; Diwadkar \& McNamara, 1997; Easton \& Sholl, 1995; Levine, Jankovic, \& Palij, 1982; Mou \& McNamara, 2002; Presson \& Montello, 1994; Richardson, Montello, \& Hegarty, 1999, map \& virtual-walk conditions; Rieser, 1989; Rieser, Guth, \& Hill, 1986; Roskos-Ewoldsen et al., 1998; Shelton \& McNamara, 1997, 2001a, 2001b, 2001c; Sholl \& Nolin, 1997, Exps. 1, 2, \& 5; Simons \& Wang, 1998). A consistent finding has been that performance is orientation dependent. In most of those studies, orientation dependence took the form of better performance on familiar views and orientations than on unfamiliar views and orientations; in Mou and McNamara's (2002) experiments, performance was better on orientations aligned with the intrinsic axis of learning than on other orientations.

Orientation independent performance has been observed, however, in several published studies (Evans \& Pezdek, 1980; Presson, DeLange, \& Hazelrigg, 1989; Presson \& Hazelrigg, 1984; Richardson et al., 1999, real-walk condition; Sholl \& Nolin, 1997, Exps. 3 \& 4). In a now classical study, Evans and Pezdek (1980) reported evidence of orientation independence in memory of a large-scale environment. Participants were shown sets of three building names, which were selected from the Cal State-San Bernardino campus, and had to decide whether or not the buildings were arranged in the correct spatial configuration. Incorrect triads were mirror images of correct triads. Participants in one experiment were students at the university who presumably learned the locations of buildings naturally via navigation; participants in another experiment were students at another university who had memorized a map of the Cal State-San Bernardino campus. The independent variable was the angular rotation of the test stimulus relative to the canonical vertical defined by the map. For students who had learned the map, the familiar upright views of the stimuli were recognized fastest, and the difficulty of recognizing unfamiliar, rotated stimuli was a linear function of angular rotation (e.g., Shepard \& Metzler, 1971). However, for students who had learned the campus naturally, there was no such relation: Response times were roughly the same at all angles of rotation. An analysis of individual participants' data revealed no linear trends even when alternative canonical orientations were considered.

To our knowledge, Evans and Pezdek's (1980) experiments have never been replicated. One explanation for the pattern of results is that students who learned the campus experienced it from many points of view and orientations, whereas students 
who learned the map only experienced the map in one orientation. Recent evidence indicates, however, that learning a large-scale environment from several orientations is not sufficient to produce an orientation independent representation. McNamara, Rump, and Werner (in press) had student participants learn the locations of eight objects in an unfamiliar city park by walking through the park on one of two prescribed paths, which encircled a large rectangular building (a full-scale replica the Parthenon in Athens, Greece). The aligned path was oriented with the building; the misaligned path was rotated by $45^{\circ}$ (see Figure 5). Participants walked the path twice, and spent about 30 minutes learning the locations of the objects. They were then driven back to the laboratory, and made judgments of relative direction using their memories. As shown in Figure 6, pointing accuracy was higher in the aligned than in the misaligned path group, and the patterns of results differed: In the aligned condition, accuracy was relatively high for imagined headings parallel to legs of the path $\left(0^{\circ}, 90^{\circ}, 180^{\circ}, 270^{\circ}\right)$ and for an imagined heading oriented toward a nearby lake, a salient landmark $\left(225^{\circ}\right)$. In the misaligned condition, pointing accuracy was highest for the imagined heading oriented toward the lake (a heading that was familiar), and decreased monotonically with angular distance. For both groups, though, performance was orientation dependent; there was no evidence that participants were

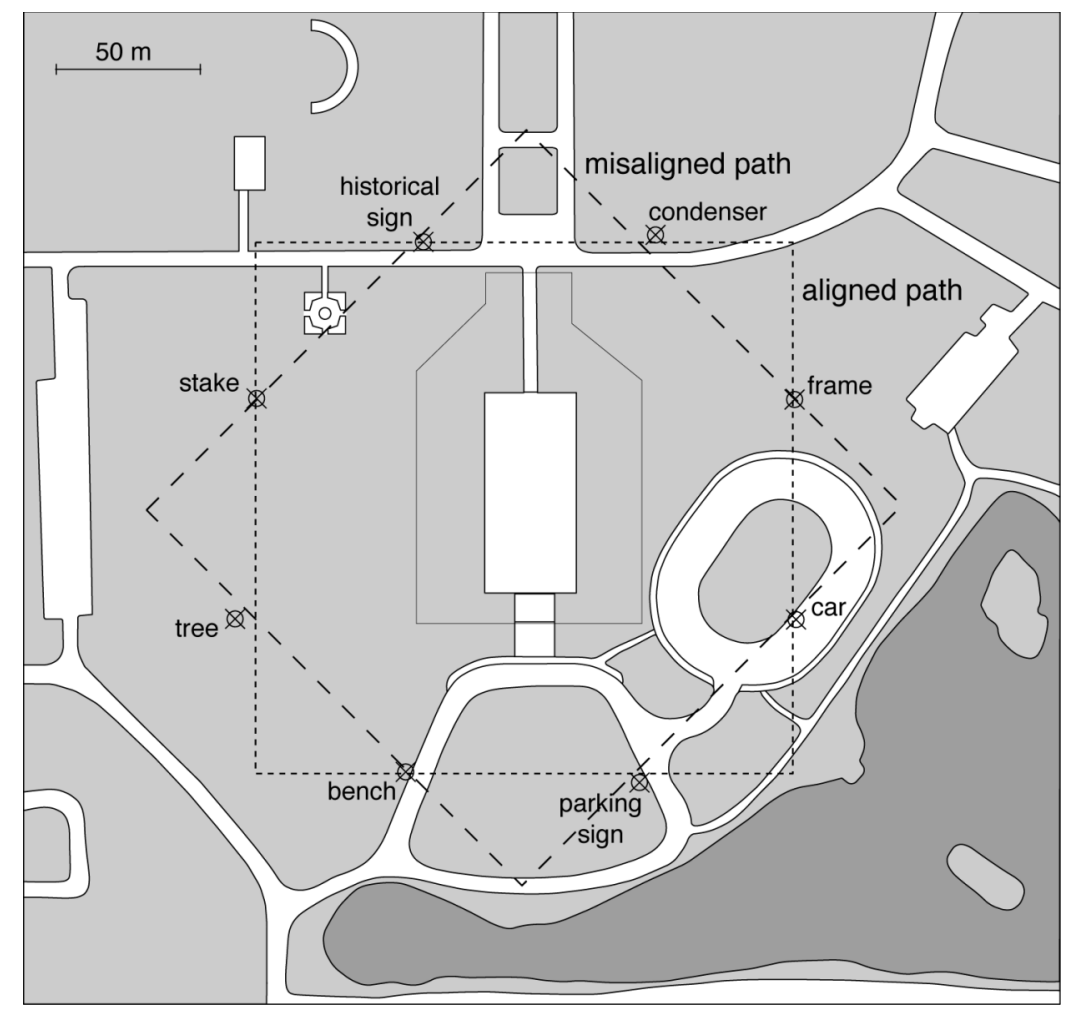

Figure 5. Map of the park and paths in McNamara, Rump, and Werner's (in press) experiment. The white rectangle in the center is the Parthenon. Dark shaded area in lower right is the lake. 


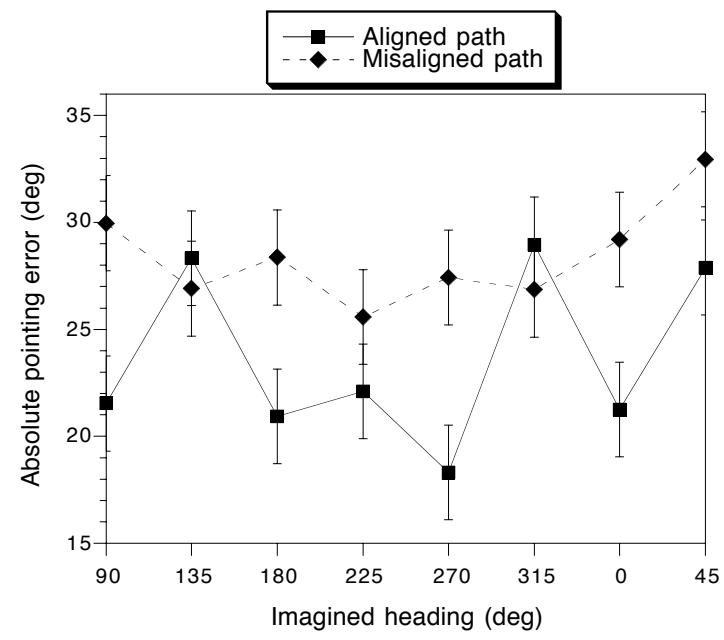

Figure 6. Angular error in judgments of relative direction as a function of imagined heading and path. Subjects learned the locations of 8 objects in the park by walking either the aligned path or the misaligned path (see Figure 5). Data are plotted to emphasize the symmetry around the heading of $225^{\circ}$. Error bars are confidence intervals corresponding to \pm 1 SEM as estimated from the ANOVA.

able to construct view-invariant representations of the spatial structure of the park after experiencing it from four orientations.

In another influential line of research, Presson and his colleagues (Presson et al., 1989; Presson \& Hazelrigg, 1984) obtained evidence that orientation dependence was modulated by layout size. Participants learned 4-point paths from a single perspective. These paths were small (e.g., $40 \mathrm{~cm} \mathrm{X} 40 \mathrm{~cm}$ ) or large (e.g., $4 \mathrm{~m} \mathrm{X} 4 \mathrm{~m}$ ). After learning a layout, participants made judgments of relative direction using their memories of the layout. Imagined headings were aligned or contra-aligned with the original viewing perspective. The experiments showed that layout size was the only consistent predictor of the relative difficulty of aligned and contra-aligned judgments. When participants learned small layouts, aligned judgments were more accurate than contra-aligned judgments, but when they learned large layouts, the difference in accuracy was reduced or eliminated. This interaction occurred even though participants viewed small and large layouts from a single perspective.

Roskos-Ewoldsen et al. (1998) attempted to replicate the learning and the test conditions used by Presson and his colleagues (Presson et al., 1989; Presson \& Hazelrigg, 1984), and yet still obtained orientation dependent performance. We learned after conducting the experiments that there were important differences in how participants were tested. Roskos-Ewoldsen et al. discussed these differences at length, and concluded that participants in Presson's experiments might have been able to update their mental representations when they were tested (e.g., Rieser, 1989).

Sholl and Nolin (1997) also attempted to replicate Presson, DeLange, and Hazelrigg's (1989) findings, and for the most part, were unable to do so. However, Sholl and Nolin were able to obtain orientation independent performance in one 
combination of conditions, namely, when participants learned the 4-point paths from a low viewing angle (e.g., while seated) and were tested in a condition in which their physical location and facing direction at the time they made their pointing judgment matched those specified in the judgment of relative direction. Unfortunately, these same learning and test conditions produced orientation dependent performance in experiments conducted by Mou and McNamara (2001), although their participants learned more complex layouts (seven objects distributed on the floor of a large room as opposed to 4-point paths).

Finally, Richardson, Montello, and Hegarty (1999) had participants learn the interior hallways of a large building by walking through the building, by navigating a desktop virtual environment, or by learning a map. Afterwards, participants engaged in several tasks, including pointing to target locations from imagined and actual locations in the building. Orientation dependence was tested in the virtual-walk and in the real-walk conditions by comparing pointing judgments for headings aligned with the first leg of the path to pointing judgments for other headings. Aligned judgments were more accurate than misaligned judgments in the virtual-walk condition but these judgments did not differ in the real-walk condition, suggesting that real movement in the space allowed participants to form orientation independent mental representations. It is possible that if alignment were defined with respect to a different reference axis (e.g., the longest leg of the path), or different reference axes for different participants, evidence of orientation dependence might appear (e.g., Valiquette, McNamara, \& Smith, 2002).

An important feature of all of the experiments in which orientation independent performance has been observed, with the exception of the Evans and Pezdek (1980) experiments, is that only two orientation conditions were compared: In the aligned condition, the imagined heading was parallel to the learning view (e.g., in Figure 1, "Imagine you are at the book, facing the wood; point to the clock"), and in the contraaligned condition, the imagined heading differed by $180^{\circ}$ from the learning view (e.g., "Imagine you are at the wood, facing the book; point to the clock"). This fact may be important because performance in judgments of relative direction for the imagined heading of $180^{\circ}$ is often much better than performance for other novel headings, and can be nearly as good as that for the learning view (see, e.g., Figure 4). The cause of this effect is not clear, but it is possible that, for as yet unknown reasons, participants sometimes represent, at least partially, the spatial structure of the layout in the contraaligned direction. It is also possible that participants are able to capitalize on selfsimilarity under rotations of $180^{\circ}$ under certain conditions (e.g., Vetter, Poggio, \& Bülthoff, 1994). In our opinion, investigations of the orientation dependence of spatial memories are at a distinct disadvantage if only aligned and contra-aligned conditions are compared.

In summary, there may be conditions in which people are able to form orientation independent spatial representations but these situations seem to be the exception rather than the rule; in addition, attempts to replicate some of these findings have not been successful. In our opinion, the balance of evidence indicates that spatial memories are orientation-dependent. 


\section{One Representation or Two?}

Recent experiments conducted in our laboratory suggest that at least two independent representations may be formed when participants learn a spatial layout visually. One of these representations seems to preserve interobject spatial relations, and is used to make judgments of relative direction, whereas the other is a visual memory of the layout, and supports scene recognition.

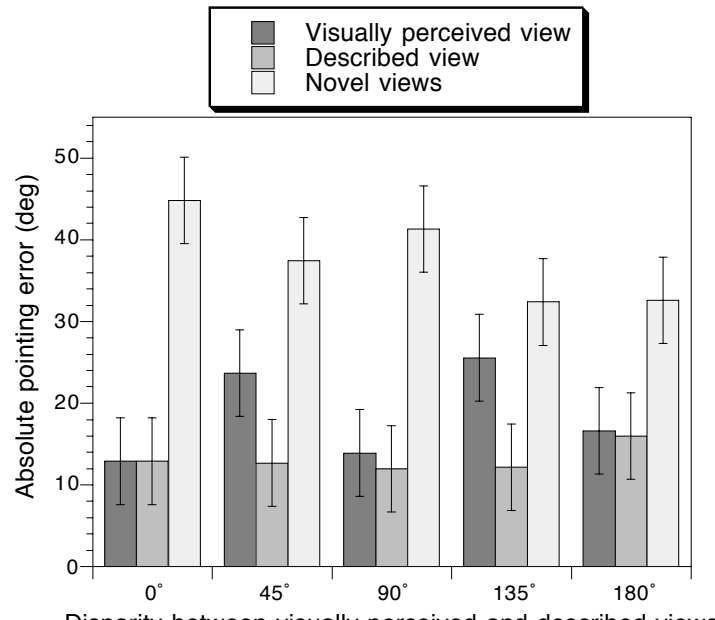

Figure 7. Angular error in judgments of relative direction in Shelton and McNamara's (2001a) experiment. Error bars are confidence intervals corresponding to \pm 1 SEM as estimated from the ANOVA.

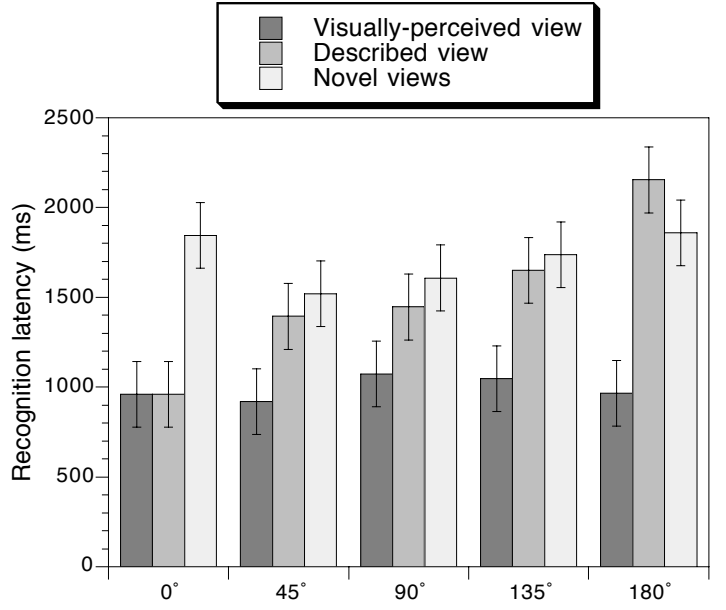

Disparity between visually-perceived and described views

Figure 8. Response latency in visual scene recognition in Shelton and McNamara's (2001a) experiment. Error bars are confidence intervals corresponding to \pm 1 SEM as estimated from the ANOVA. 
One of these experiments was an investigation of spatial perspective taking (Shelton \& McNamara, 2001a). One participant (the director) viewed a display of objects from a single perspective and described the display to a second participant (the matcher) from a perspective that differed by $0^{\circ}, 45^{\circ}, 90^{\circ}, 135^{\circ}$, or $180^{\circ}$ from the viewing perspective (e.g., Schober, 1993). The matcher's task was to reconstruct the layout from the director's description. The two were separated by a barrier that prevented visual contact. After they had finished, the director's memory for the spatial layout was tested using judgments of relative direction, old-new scene recognition (e.g., Diwadkar \& McNamara, 1997), and map drawing. We were particularly interested in the effects of describing the layout from a nonegocentric perspective on the director's memory of the layout. Angular error in judgments of relative direction is reproduced in Figure 7. These results indicated that the described view was represented in memory at least as well as, and in two conditions (viz., disparities of $45^{\circ}$ and $135^{\circ}$ ) better than, the visually perceived view. By contrast, the results from scene recognition (Figure 8) indicated that only the visually perceived view was represented in memory; the described view was recognized no faster and no more accurately than were novel views (which had been neither seen nor described). Scene recognition is a visual task, so it is not surprising that participants could not recognize the "unseen" view as fast as they could recognize the visually perceived view. It is, however, intriguing that the described view, which clearly showed a benefit in judgments of relative direction, appeared to be no different from a novel view during scene recognition.

Additional evidence of multiple spatial representations can be found in an experiment just completed in our laboratory. The learning phase of this experiment replicated that of Shelton and McNamara's (2001b) Experiment 3, which was summarized earlier in this chapter (Figures 1 and 2). After learning, participants took part in two tasks: Old-new scene recognition, in which participants had to discriminate pictures of the layout, regardless of point of view, from pictures of the same objects in different spatial configurations; and judgments of relative direction. The results of judgments of relative direction (see Figure 9) largely replicated our original findings (see Figure 2), and indicated that the aligned view was mentally represented but the misaligned view was not: Performance for the familiar heading of $135^{\circ}$ was worse than performance for the familiar heading of $0^{\circ}$, and not statistically better than performance for unfamiliar headings. As discussed earlier, we attribute the savings at headings of $90^{\circ}, 180^{\circ}$, and $270^{\circ}$ to partial representation of spatial relations along directions orthogonal and opposite to the primary intrinsic direction. The results from scene recognition (see Figure 10), however, showed that views of $0^{\circ}$ and $135^{\circ}$ were recognized equally well, and better than views from novel perspectives. This pattern indicated that both views were mentally represented. There were no effects of order of learning (0-135 vs. 135-0) in either task, and both graphs collapse across this variable.

\section{Summary and Prospectus}

Our primary goal in this chapter was to summarize a new theory of spatial memory. This theory, which is still in its infancy, attempts to explain how the locations of objects in the environment are represented in memory. 
According to the theory, when people learn a new environment, they represent the locations of objects in terms of a reference system intrinsic to the layout itself. Axes intrinsic to the collection of objects are selected and used to represent location and orientation. These axes are chosen on the basis of egocentric experience (including verbal instructions), spatial and nonspatial properties of the objects, and cues in the surrounding environment. We view this process as being analogous to identifying the top of a figure; in effect, conceptual "north" (and perhaps, east, west, $\&$ south) is created at the time of learning. Recent findings also suggest, however,

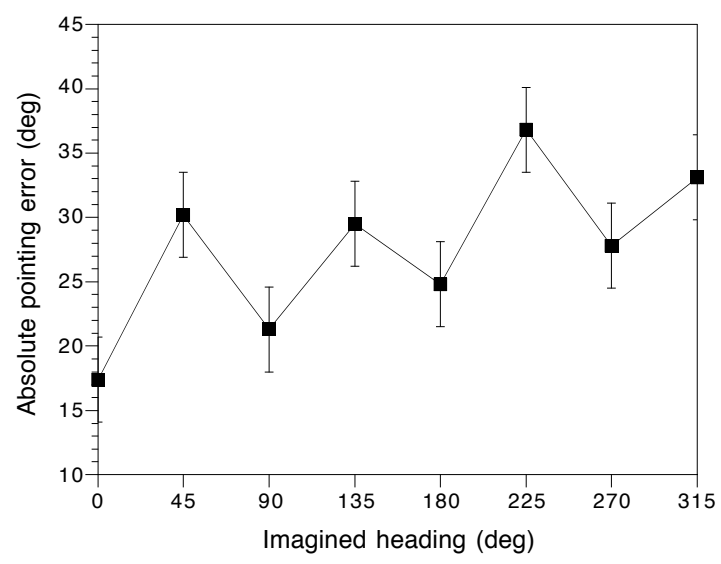

Figure 9. Angular error in judgments of relative direction as a function of imagined heading. Subjects learned an aligned view $\left(0^{\circ}\right)$ and a misaligned view $\left(135^{\circ}\right)$ of layouts similar to the one illustrated in Figure 1. Error bars are confidence intervals corresponding to \pm 1 SEM as estimated from the ANOVA.

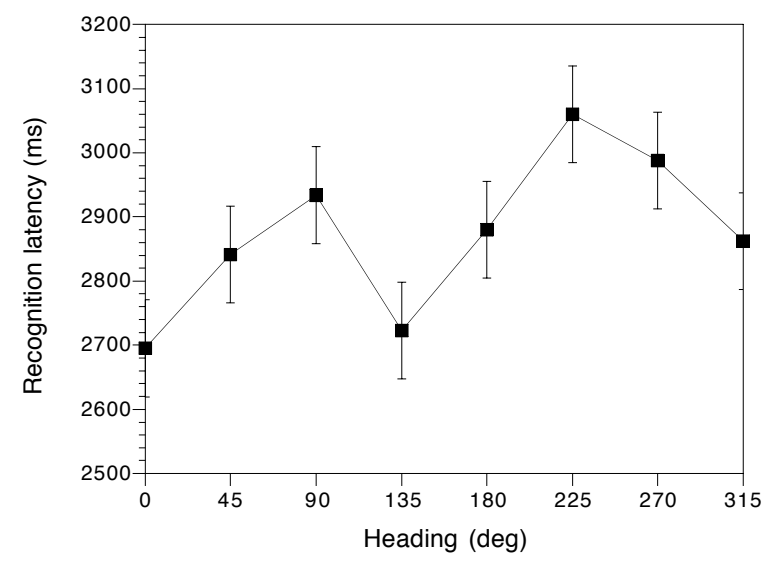

Figure 10. Response latency in visual scene recognition as a function of heading. Subjects learned an aligned view $\left(0^{\circ}\right)$ and a misaligned view $\left(135^{\circ}\right)$ of layouts similar to the one illustrated in Figure 1. Error bars are confidence intervals corresponding to \pm 1 SEM as estimated from the ANOVA. 
that visual memories of familiar views are stored, regardless of their alignment with environmental reference systems. The relationship between these two spatial representations is unknown at this time.

This theory makes the strong claim that spatial memories are composed primarily of object-to-object spatial relations, and therefore are allocentric. This claim conflicts with several recent proposals that spatial memories are primarily egocentric (e.g., Shelton \& McNamara, 1997; Wang, 1999). Egocentric self-to-object spatial relations must be represented, at least at the perceptual level, for the purpose of guiding action in the environment (e.g., Anderson, 1999). It is an open question, however, whether egocentric spatial relations are represented in long-term memory. In principle, the spatial information needed for spatially directed motor activity could be computed from object-to-object spatial relations. Such a division of labor in spatial representation and processing between a transient egocentric system and a more permanent allocentric system bears strong resemblance to Milner and Goodale's (1995) account of dorsal and ventral streams of visual processing in the primate brain.

As people move through an environment, they must continuously update their location and orientation with respect to stable elements of the landscape to avoid getting lost or disoriented. Investigations of the ability to update with respect to a previously experienced collection of objects indicate that updating is of high fidelity and automatic, in the sense that it cannot be ignored (e.g., Farrell \& Robertson, 1998; Rieser, 1989). Evidence from our laboratory indicates that the object-to-object system is not updated. For instance, if participants had updated in Shelton and McNamara's (2001b) cylindrical room experiment, one would expect performance to have been best on the heading parallel to the last study view, or perhaps on headings parallel to each of the three study views. In fact, spatial relations were retrieved efficiently only from the first study view. If the object-to-object system is not updated during locomotion, what is updated? Sholl and Nolin (1997) and Wang (1999) have suggested that egocentric self-to-object spatial relations are updated continuously as people move through an environment. It is also possible, however, that people update their position and orientation with respect to the same reference system used to represent the spatial structure of the environment, in effect treating their bodies as just another object in the space. Investigations of these questions should lead to a better understanding of how remembered spatial relations are used to guide action in space.

\section{References}

Anderson, R. A. (1999). Multimodal integration for the representation of space in the posterior parietal cortex. In N. Burgess, K. J. Jeffery, \& J. O'Keefe (Eds.), The hippocampal and parietal foundations of spatial cognition (pp. 90-103). Oxford: Oxford University Press.

Bryant, D. J., \& Tversky, B. (1999). Mental representations of perspective and spatial relations from diagrams and models. Journal of Experimental Psychology: Learning, Memory, and Cognition, 25, 137-156.

Christou, C. G., \& Bülthoff, H. H. (1999). View dependence in scene recognition after active learning. Memory \& Cognition, 27, 996-1007.

Diwadkar, V. A., \& McNamara, T. P. (1997). Viewpoint dependence in scene recognition. Psychological Science, 8, 302-307. 
Easton, R. D., \& Sholl, M. J. (1995). Object-array structure, frames of reference, and retrieval of spatial knowledge. Journal of Experimental Psychology: Learning, Memory, and Cognition, 21, 483-500.

Evans, G. W., \& Pezdek, K. (1980). Cognitive mapping: Knowledge of real-world distance and location information. Journal of Experimental Psychology: Human Learning and Memory, $6,13-24$.

Farrell, M. J., \& Robertson, I. H. (1998). Mental rotation and the automatic updating of bodycentered spatial relationships. Journal of Experimental Psychology: Learning, Memory, and Cognition, 24, 227-233.

Franklin, N., \& Tversky, B. (1990). Searching imagined environments. Journal of Experimental Psychology: General, 119, 63-76.

Friedman, A., \& Hall, D. L. (1996). The importance of being upright: Use of environmental and viewer-centered reference frames in shape discriminations of novel three-dimensional objects. Memory \& Cognition, 24, 285-295.

Hermer, L., \& Spelke, E. S. (1994). A geometric process for spatial reorientation in young children. Nature, 370, 57-59.

Huttenlocher, J., Hedges, L. V., \& Duncan, S. (1991). Categories and particulars: Prototype effects in estimating spatial location. Psychological Review, 98, 352-376.

Lansdale, M. W. (1998). Modeling memory for absolute location. Psychological Review, 105, 351-378.

Learmonth, A. E., Newcombe, N. S., \& Huttenlocher, J. (2001). Toddlers' use of metric information and landmarks to reorient. Journal of Experimental Child Psychology, 80, 225244.

Levine, M., Jankovic, I. N., \& Palij, M. (1982). Principles of spatial problem solving. Journal of Experimental Psychology: General, 111, 157-175.

Levinson, S. C. (1996). Frames of reference and Molyneaux's question: Crosslinguistic evidence. In P. Bloom, M. A. Peterson, L. Nadel, \& M. F. Garrett (Eds.), Language and space (pp. 109-169). Cambridge, MA: MIT Press.

McMullen, P. A., \& Jolicoeur, P. (1990). The spatial frame of reference in object naming and discrimination of left-right reflections. Memory \& Cognition, 18, 99-115.

McNamara, T. P., Rump, B., \& Werner, S. (in press). Egocentric and geocentric frames of reference in memory of large-scale space. Psychonomic Bulletin \& Review.

Milner, A. D., \& Goodale, M. A. (1995). The visual brain in action. Oxford: Oxford University Press.

Montello, D. R. (1991). Spatial orientation and the angularity of urban routes: A field study. Environment and Behavior, 23, 47-69.

Mou, W., \& McNamara, T. P. (2001). Spatial memory and spatial updating. Unpublished manuscript.

Mou, W., \& McNamara, T. P. (2002). Intrinsic frames of reference in spatial memory. Journal of Experimental Psychology: Learning, Memory, and Cognition, 28, 162-170.

Palmer, S. E. (1989). Reference frames in the perception of shape and orientation. In B. E. Shepp \& S. Ballesteros (Eds.), Object perception: Structure and process (pp. 121-163). Hillsdale, NJ: Erlbaum.

Presson, C. C., DeLange, N., \& Hazelrigg, M. D. (1989). Orientation specificity in spatial memory: What makes a path different from a map of the path? Journal of Experimental Psychology: Learning, Memory, and Cognition, 15, 887-897.

Presson, C. C., \& Hazelrigg, M. D. (1984). Building spatial representations through primary and secondary learning. Journal of Experimental Psychology: Learning, Memory, and Cognition, 10, 716-722.

Presson, C. C., \& Montello, D. R. (1994). Updating after rotational and translational body movements: Coordinate structure of perspective space. Perception, 23, 1447-1455. 
Richardson, A. E., Montello, D. R., \& Hegarty, M. (1999). Spatial knowledge acquisition from maps and from navigation in real and virtual environments. Memory \& Cognition, 27, 741750.

Rieser, J. J. (1989). Access to knowledge of spatial structure at novel points of observation. Journal of Experimental Psychology: Learning, Memory, and Cognition, 15, 1157-1165.

Rieser, J. J., Guth, D. A., \& Hill, E. W. (1986). Sensitivity to perspective structure while walking without vision. Perception, 15, 173-188.

Rock, I. (1956). The orientation of forms on the retina and in the environment. American Journal of Psychology, 69, 513-528.

Rock, I. (1973). Orientation and form. New York: Academic Press.

Roskos-Ewoldsen, B., McNamara, T. P., Shelton, A. L., \& Carr, W. (1998). Mental representations of large and small spatial layouts are orientation dependent. Journal of Experimental Psychology: Learning, Memory, and Cognition, 24, 215-226.

Schober, M. F. (1993). Spatial perspective-taking in conversation. Cognition, 47, 1-24.

Shelton, A. L., \& McNamara, T. P. (1997). Multiple views of spatial memory. Psychonomic Bulletin \& Review, 4, 102-106.

Shelton, A. L., \& McNamara, T. P. (2001a). Spatial memory and perspective taking. Unpublished manuscript.

Shelton, A. L., \& McNamara, T. P. (2001b). Systems of spatial reference in human memory. Cognitive Psychology, 43, 274-310.

Shelton, A. L., \& McNamara, T. P. (2001c). Visual memories from nonvisual experiences. Psychological Science, 12, 343-347.

Shepard, R. N., \& Metzler, J. (1971). Mental rotation of three-dimensional objects. Science, 171, 701-703.

Sholl, M. J. (1987). Cognitive maps as orienting schemata. Journal of Experimental Psychology: Learning, Memory, and Cognition, 13, 615-628.

Sholl, M. J., \& Nolin, T. L. (1997). Orientation specificity in representations of place. Journal of Experimental Psychology: Learning, Memory, and Cognition, 23, 1494-1507.

Simons, D. J., \& Wang, R. F. (1998). Perceiving real-world viewpoint changes. Psychological Science, 9, 315-320.

Tversky, B. (1981). Distortions in memory for maps. Cognitive Psychology, 13, 407-433.

Valiquette, C. M., McNamara, T. P., \& Smith, K. (2002). Locomotion, incidental learning, and the orientation dependence of spatial memory. Unpublished manuscript.

Vetter, T., Poggio, T., \& Bülthoff, H. H. (1994). The importance of symmetry and virtual views in three-dimensional object recognition. Current Biology, 4, 18-23.

Wang, R. F. (1999). Representing a stable environment by egocentric updating and invariant representations. Spatial Cognition and Computation, 1, 431-445.

Werner, S., \& Schmidt, K. (1999). Environmental reference systems for large scale spaces. Spatial Cognition and Computation, 1, 447-473. 